\title{
Qualitative assessment of the dental health services provided at a dental school in Kerman, Iran
}

\section{Maryam Rad(a) \\ Jahangir Haghani(b) \\ Arash Shahravan (c) \\ Ali Khosravifar(d)}

(a) Assistant professor, Department of Oral Medicine; (b) Assistant professor, Department of Radiology; ${ }^{(c)}$ Assistant professor, Member of the Kerman Oral and Dental Diseases Research Center - Dental School, Medical University of Kerman, Kerman, Iran.

(d) DDS.
Corresponding author:

Maryam Rad

Shafa Street, Department of Oral Medicine, School of Dentistry

Medical University of Kerman

Kerman - Iran

E-mail:Rad_1152@yahoo.com

Received for publication on Jun 21, 2008

Accepted for publication on Feb 20, 2009

\begin{abstract}
Increasing the quality of the services provided in a Dental School can raise the satisfaction level of patients and consequently increase the level of their oral health. This study was conducted to evaluate the quality of dental care and services provided to patients referred to a Dental School in Kerman, Iran. In this qualitative study, face-to-face, in-depth interviews were conducted with 41 participants $[25$ patients $(\mathrm{P})$, 5 nurses (N), 6 dental academic staff (AS), and 5 dental students (S)]. Then, the interviews were transcribed and analyzed, using content analysis of data. Data analysis in qualitative research involves breaking down the data and searching for codes and categories that are then reassembled to form themes. Both positive and negative themes emerged. Positive themes included: good infection control, service accessibility, patient appointments and visits were not assigned on merit, precise examinations, and comprehensive treatment plans. Negative themes included: long wait time, lack of options to pass waiting time, such as newspapers and television, an insufficient number of nurses, and not enough professors for supervision. In addition, the results of this study show that the patients and dental staff have high expectations in relation to dental services, and that implementation of these expectations would increase the overall satisfaction with and the quality of the level of services. Finally, some recommendations for improving services in the Kerman Dental School were given to the managing team of the Dental School.
\end{abstract}

Descriptors: Health care quality, access, and evaluation; Dental schools; Patient satisfaction. 


\section{Introduction}

The quality of various services in dental schools has become a major issue. ${ }^{1}$ Obviously, improving the quality of services can increase the satisfaction level of patients. Patient satisfaction is an important factor that influences both the treatment mode and the relationship with the dentist. ${ }^{2}$ Recent issues in dentistry, including patient expectations of dental services and barriers to access of dental services, raise important questions that cannot be completely answered by a quantitative study, but may best be studied by qualitative research. ${ }^{3}$ Qualitative studies can explore the complexity of the issue and give meaning to the social phenomena related to the values of dental services and experiences in health care. ${ }^{4,5}$ Furthermore, this form of study can answer important, basic questions, such as why this happens, what does it mean, what factors influence the outcome, what are the barriers to access, and what do the facilities lack? ${ }^{6}$ This kind of research will explore new topics and develop deeper understanding in the treatment and satisfaction of the patients. ${ }^{7,8}$

No previous studies have investigated what patients expect from dental services and what the problems are in dental schools in Iran. Therefore, this study was conducted in order to investigate and evaluate the quality of dental care and services among the patients referred to a Dental School in Kerman, Iran.

\section{Material and Methods}

This study follows an action research model, within the domain of qualitative research. In this study, we used semi-structured, face-to-face, in-detail interviews and, in addition, six focus group discussions. All interviews and group discussions were conducted by the four authors, and were continued until data saturation had been attained ( 25 patients, 5 students, 5 nurses and 6 dental academic staff). The interviews and group discussions were recorded on cassette tapes, and the recordings were followed by analysis. Data analysis in qualitative research involves breaking down the data into codes and categories. ${ }^{9}$ The analyzed data was categorized and the number of respondents in each category was specified. In addition, to more objectively verify the ac- curacy of the codes taken from the interviews, each of the codes was revised by the interviewers so that the researchers could have a better and more precise understanding of the answers the patients gave to the questions.

\section{Results}

Of the 41 interviews, 25 were with patients of differing occupational status, who were selected from a variety of ages, and chosen equally from male or female patients.

The main causes for patient satisfaction included:

- The site and location of the Dental School.

- Infection control and sterilization.

- Lack of discrimination.

- A comprehensive treatment procedure.

- Very proficient examinations and quick responses to requests for the cost of laboratory tests in the department of oral medicine.

Specific barriers to receiving dental services emerged as major themes, which included:

- Quickly obtaining an appointment (especially in the endodontics and prosthodontics departments).

- Lack of centers associated with the dental schools in other parts of the city.

- Lack of good insurance for dental treatment.

- Delay in attending to patients, causing the patients' time to be wasted while in the Dental School.

- Incomplete treatment with insufficient timing.

- Insufficient number of nurses, and not enough professors for supervision.

- Long course of treatment, especially in the endodontics department.

- Lack of coordination between departments.

- Lack of dental magazines and posters in the waiting room.

- Lack of options to pass times in the waiting rooms (TV, newspapers, journals, buffet, etc.).

- Weekly treatment appointments, especially in the periodontics department.

- Lack of radiography equipment in the prosthodontics department.

- Lack of confidence in the quality of dental materials.

The main causes for dissatisfaction among the 
academic staff, nurses, and students included:

- Keeping patients waiting.

- Inadequate comfortable facilities for the patients.

- Inadequate comfortable facilities for the dental staff and students.

- Inadequate dental academic staff and nurses, especially in the prosthodontics department.

- Lack of digital files in some departments (for example, in the prosthodontics and pediatric departments).

- Inadequately trained students and incomplete treatments.

- Lack of partitions between units.

- Inadequate materials (for example, capsules of amalgams).

- Lack of specialization in some departments.

- Need for additional education for some of the nurses and students in infection control.

\section{Discussion}

Unlike quantitative research, qualitative research considers the reasons behind various aspects of behavior. ${ }^{5}$ Qualitative studies investigate the why and how of decision making, as compared to the what, where, and when of quantitative research. Hence, the need is for smaller but focused samples rather than large random samples, because qualitative research categorizes data into patterns as the primary basis for organizing and reporting results. ${ }^{6}$ We undertook this study considering this point and the fact that there is still a lack of qualitative research about the quality of the services provided in Iran's dental schools and other countries, in spite of the existence of many qualitative research studies in dentistry in the current decades (for example, the qualitative assessment of dental care among the elderly population in the United States; ${ }^{3}$ patient expectations of emergency dental services in Australia; ${ }^{10}$ the effectiveness of out-of-hours dental services in the UK. ${ }^{11}$ ). There are also not many studies assessing the quality of services provided in dental schools. Therefore, the results of this study are not completely comparable with studies of the quality of services provided in other dental schools.

In this study, the main causes for dissatisfaction included:
- Difficulty in making appointments (10P, 4AS, $5 \mathrm{~S}, 5 \mathrm{~N})$.

This barrier might be removed by establishing centers affiliated with the dental school and providing emergency dental services.

- Inadequate comfortable facilities and lack of dental magazines and posters (5P, 4AS, 4N, 5S). The dental school needs to mobilize, and supply dental magazines, posters, and so on, that can effectively increase patients' knowledge about oral and dental medicine and methods of preventing dental diseases.

- Inadequate number of nurses and academic staff (6AS, 5N, 5S).

There is a need to increase the recruitment of dental staff.

- Lack of medical insurance (10P, 2S).

There is a need to cooperate with insurance organizations in order to increase the satisfaction level of all patients. (Also, see notes that follow.) In this study, ten patients and two students who were interviewed expressed their belief that dental treatments are too expensive. Hill and colleagues have also shown that the cost of dental treatment was given as a reason for non-attendance or postponing a trip to the dentist. ${ }^{2}$ Also, Liddell and colleagues have shown that the costs of dental care may be a barrier to the uptake of dental services. ${ }^{11}$

These two studies evaluated personal dental services. However, in our study other patients were satisfied with dental fees, and expressed their belief that the cost of dental care in the Dental School may motivate an increase in dental services in the school (compared with private dental care units).

Although in this study all patients were satisfied with infection control, the medical staff believes that the Head of the Dental School should plan a continuous education program for infection control.

Many studies have shown that access to dental assistance, ${ }^{12,13}$ costs of dental treatment, ${ }^{14,15}$ and the availability of dental insurance, ${ }^{12,13,16}$ play an important role in the satisfaction of patients. A study in Australia about access to dental care found that patients with dental insurance are more likely to have visited a dentist within the last two years. ${ }^{12}$ In Lithuania, Puriene et al. ${ }^{14}$ (2008) showed that patients 
who preferred to visit public institutions required less expensive treatment. In Germany, a dental practice survey, with patient evaluation of dental care, showed the most critical evaluations were given for waiting time, costs of the dental treatment for the patient, and the range of magazines and printed information in the waiting room. ${ }^{15}$

In addition to the above comments and notes, to increase the satisfaction level of patients, we suggest that heads of the dental school in Kerman should do the following:

- Increase the number of supervising professors during treatment procedures by the dental staff.

- Perform complete treatments during the term of the discipline in which dental care is being provided.

- Coordinate departments for better treatment procedures.

- Plan programs for providing the best materials and equipment.

\section{References}

1. Meadows LM, Verdi AJ, Crabtree BF. Keeping up appearances: using qualitative research to enhance knowledge of dental practice. J Dent Educ. 2003 Sep;67(9):981-90.

2. Hill KB, White DA, Morris AJ, Hall AC, Goodwin N, Burke FJ. National evaluation of personal dental services: a qualitative investigation into patients' perceptions of dental services. Br Dent J. 2003 Dec 6;195(11):654-6.

3. Robbertz AA, Lauf Jr RC, Rupp RL, Alexander DC. A qualitative assessment of dental care access and utilization among the older adult population in the United States. Gen Dent. 2006 Sep-Oct;54(5):361-5; quiz 366-8.

4. Sackett DL, Wennberg JE. Choosing the best research design for each question. BMJ. 1997 Dec 20-27;315(7123):1636.

5. Bailey J. First steps in qualitative data analysis: transcribing. Fam Pract. 2008 Apr; 25(2):127-31.

6. Hendricson B. It all starts with questions. J Dent Educ. 2003 Sep;67(9):965-9.

7. Hilton IV, Stephen S, Barker JC, Weintraub JA. Cultural factors and children's oral health care: a qualitative study of carers of young children. Community Dent Oral Epidemiol. 2007 Dec;35(6):429-38.

8. Wong LP. Focus group discussion: a tool for health and medical research. Singapore Med J. 2008 Mar;49(3):256-60; quiz 261.

\section{Conclusion}

In this study, the main causes of patient satisfaction included: good infection control, service accessibility, patient appointments and visits were not assigned on merit, precise examinations, and comprehensive treatment plans. The main causes of dissatisfaction included: long wait time, lack of options to pass waiting time, such as newspapers and television, an insufficient number of nurses, and not enough professors for supervision. The results of this study show that the patients and dental staff have high expectations in regards to dental services, and that implementation of these expectations would increase the overall satisfaction with and the quality of the level of services.

\section{Acknowledgements}

The authors wish to thank The Research Center of the Kerman Medical Science for their financial support.

9. Anderson R. Patient expectations of emergency dental services: a qualitative interview study. Br Dent J. 2004 Sep 25;197(6):331-4; discussion 323.

10. Anderson R, Thomas DW, Phillips CJ. The effectiveness of out-of-hours dental services: II. patient satisfaction. Br Dent J. 2005;198:151-6; discussion 146.

11. Liddell A, May B. Some characteristics of regular and irregular attenders for dental check-ups. Br J Clin Psychol. 1984;23(Pt 1):19-26

12. Ellershaw A. Oral health and access to dental care in Australia - comparisons by level of education. Aust Dent J. 2006 Dec;51(4):342-5.

13. Kruka ME, Freedman LP. Assessing health system performance in developing countries: A review of the literature. Health Policy. 2008;85:263-76.

14. Pūriene A, Petrauskiene J, Balciūniene I, Janulyte V, Kutkauskiene J, Musteikyte M. [Private or public dental care? Patients' perception and experience in Lithuania] [Article in Lithuanian]. Medicina (Kaunas). 2008;44(10):805-11.

15. Klingenberg A, Walther W, Dörfer CE, Szecsenyi J. Patient evaluation of dental care. Results of a written patient survey in dental practices. Gesundheitswesen. 2008 Aug-Sep;70(89):525-31.

16. Kiyak HA, Reichmuth M. Barriers to and Enablers of Older Adults' Use of Dental services. J Dent Educ. 2005;69(9):97586. 